\title{
On the primeness of near-rings
}

Khalid H. Al-Shaalan

Department of Mathematics, College of Science, King Saud University, Riyadh, Saudi Arabia

\begin{abstract}
In this paper, we study the different kinds of the primeness on the class of near-rings and we give new characterizations for them. For that purpose, we introduce new concepts called set-divisors, ideal-divisors, etc. and we give equivalent statements for 3-primeness which make 3-primeness looks like the forms of the other kinds of primeness. Also, we introduce a new different kind of primeness in near-rings called K-primeness which lies between 3-primeness and e-primeness. After that, we study different kinds of prime ideals in nearrings and find a connection between them and new concepts called set-attractors, ideal-attractors, etc. to make new characterizations for them. Also, we introduce a new different kind of prime ideals in near-rings called K-prime ideals.
\end{abstract}

Keywords Near-rings, Rings, Primeness, Prime ideals

Paper type Original Article

\section{Introduction}

We say that $R$ is a right (left) near-ring if $(R,+)$ is a group, $(R, \cdot)$ is a semigroup and $R$ satisfies the right (left) distributive law. Throughout this paper, $R$ will be a left near-ring. We say that $R$ is an abelian near-ring if $x+y=y+x$ for all $x, y \in R$ and we say that $R$ is a commutative near-ring if $x y=y x$ for all $x, y \in R$. A zero-symmetric element is an element $x \in R$ satisfying $0 x=0$. A near-ring $R$ is called a zero-symmetric near-ring, if $0 x=0$ for all $x \in R$. A constant element is an element $y \in R$ satisfying $z y=y$ for all $z \in R$. An element $x \in R$ is called a right (left) zero divisor in $R$ if there exists a non-zero element $y \in R$ such that $y x=0$ $(x y=0)$. A zero divisor is either a right or a left zero divisor. By a near-ring without zero divisors, we mean a near-ring without non-zero divisors of zero. If $A$ and $B$ are two non-empty subsets of $R$, then the product $A B$ means the set $\{a b \mid a \in A, b \in B\}$. We say that $U$ is a right (left) $R$-subgroup of $R$, if $U$ is a subgroup of $(R,+)$ satisfies $U R \subseteq U(R U \subseteq U)$. We say that $U$ is a two-sided $R$-subgroup of $R$, if $U$ is both a right and a left $R$-subgroup of $R$. We say that $I$ is a right (left) ideal of $R$, if $I$ is a normal subgroup of $(R,+)$ satisfies $(r+i) s-r s \in I$ for all $i \in I, r, s \in R(R I \subseteq I)$. We say that $I$ is an ideal of $R$ if it is both a right and a left ideal of $R$. We say that $U$ is a semigroup right (left) ideal of $R$, if $U$ is a non-empty subset of $R$ satisfies $U R \subseteq U(R U \subseteq U)$. We say that $U$ is a semigroup ideal of $R$ if it is both a semigroup right and

\section{JEL Classification - 16D25, 16N60, 16Y30}

(C) Khalid H. Al-Shaalan. Published in the Arab Journal of Mathematical Sciences. Published by Emerald Publishing Limited. This article is published under the Creative Commons Attribution (CC BY 4.0) license. Anyone may reproduce, distribute, translate and create derivative works of this article (for both commercial and non-commercial purposes), subject to full attribution to the original publication and authors. The full terms of this license may be seen at http://creativecommons.org/licences/by/4.0/legalcode

Declaration of Competing Interest: The authors declare that they have no known competing financial interests or personal relationships that could have appeared to influence the work reported in this paper.

The publisher wishes to inform readers that the article "On the primeness of near-rings" was originally published by the previous publisher of the Arab Journal of Mathematical Sciences and the pagination of this article has been subsequently changed. There has been no change to the content of the article. This change was necessary for the journal to transition from the previous publisher to the new one. The publisher sincerely apologises for any inconvenience caused. To access and cite this article, please use Al-Shaalan, K. H. (2019), "On the primeness of near-rings", Arab Journal of Mathematical Sciences, Vol. 26 No. 1/2, pp. 233-243. The original publication date for this paper was 23/12/2019. 
AJMS

$26,1 / 2$

234 left ideal of $R$ (some authors call $U$ a right (left, two-sided) $R$-subset of $R$ [8]). For any group $(G,+), M(G)$ denotes the near-ring of all maps from $G$ to $G$ with the two operations of addition and composition of maps. $M_{o}(G)$ is the zero-symmetric subnear-ring of $M(G)$ consisting of all zero preserving maps from $G$ to itself (and to make them left near-rings we should write $f(g)$ by $g f$, where $f \in M(G)$ or $M_{o}(G)$ and $\left.g \in G\right)$. A trivial zero-symmetric nearring $R$ is a zero-symmetric near-ring such that the multiplication on the group $(R,+)$ is defined by $x y=y$ and $0 y=0$ for all $x \in R-\{0\}, y \in R$. A near-field $N$ is a near-ring in which $(N-\{0\}, \cdot)$ is a group. For further information about near-rings, see [8] and [9].

In near-rings, there are five well-known kinds of primeness. We say that: $R$ is 0 -prime (the usual primeness) if, for every two ideals $I$ and $J$ of $R, I J=\{0\}$ implies $I=\{0\}$ or $J=\{0\}, R$ is 1-prime if, for every two right ideals $K$ and $L$ of $R, K L=\{0\}$ implies $K=\{0\}$ or $L=\{0\} . R$ is 2-prime if, for every two right $R$-subgroups $A$ and $B$ of $R, A B=\{0\}$ implies $A=\{0\}$ or $B=\{0\} . R$ is 3-prime if, for all $x, y \in R, x R y=\{0\}$ implies $x=0$ or $y=0$ and $R$ is equiprime (e-prime) if, for any $0 \neq a, x, y \in R, x c a=y c a$ for all $c \in R$ implies $x=y$. These five kinds of primeness are equivalent in the class of rings. But in the class of near-rings, we have: (1) $R$ is equiprime implies that $R$ is zero-symmetric 3-prime, (2) $R$ is 3-prime implies that $R$ is 2 -prime, (3) $R$ is zero-symmetric 2-prime implies that $R$ is 1-prime and (4) $R$ is 1 -prime implies that $R$ is 0 -prime. For details about these kinds and their examples and relationships see [1-3,5-7] and [10]. A near-ring (a ring) $R$ is called 3-semiprime (semiprime) if, for all $x \in R$, $x R x=\{0\}$ implies $x=0$. An ideal $P$ of $R$ is: (i) a 0-prime ideal of $R$ if for every two ideals $A$ and $B$ of $R, A B \subseteq P$ implies that $A \subseteq P$ or $B \subseteq P$, (ii) a 1-prime ideal of $R$ if for every two right ideals $A$ and $B$ of $R, A B \subseteq P$ implies that $A \subseteq P$ or $B \subseteq P$, (iii) a 2-prime ideal of $R$ if for every two right $R$-subgroups $A$ and $B$ of $R, A B \subseteq P$ implies that $A \subseteq P$ or $B \subseteq P$, (iv) a 3-prime ideal of $R$ if for $a, b \in R, a R b \subseteq P$ implies that $a \in P$ or $b \in P$, (v) an e-prime (equiprime) ideal of $R$ if for every $a \in R-P$ and $x, y \in R, x c a-y c a \in P$ for all $c \in R$ implies that $x-y \in P$. Clearly that any nearring is a $v$-prime ideal of itself, where $v \in\{0,1,2,3, e\}$. It is well-known that (ii) implies (i) and (iv) implies (iii). Also, for zero-symmetric near-rings we have (iii) implies (ii). An ideal $I$ of $R$ is called completely prime if, for $a, b \in R, a b \in I$ implies that $a \in I$ or $b \in I$. If the zero ideal is completely prime, then we say that $R$ is completely prime. Then $R$ is completely prime if and only if $R$ is without zero divisors. For more details about prime ideals, see [2,4,5] and [10].

In [1], the authors gave us a short historical view about the primeness of near-rings. We will use it and add some information to it.

Several different generalizations of primeness for rings have been introduced for nearrings. In [6], Holcombe studied three different concepts of primeness, which he called 0-prime, 1-prime and 2-prime. In [5], Groenewald obtained further results for these and introduced further notion which he called 3-primeness. In [2], Booth, Groenewald and Veldsman gave another definition, called equiprimeness, or e-primeness. In [10], Veldsman made more studies on equiprime near-rings. In [1], Booth and Groenewald gave an element-wise characterization of the radical associated with $\nu$-primeness for $v=1,2,3, e$.

In this paper we extend the idea of primeness that they did and give some new results for the primeness of near-rings. Firstly, we introduce new concepts called set-divisors, ideal divisors, etc. These concepts are generalizations of the concept of zero divisors and give another characterization of different kinds of the primeness in near-rings and hence in rings. Also, we study the 3-primeness and give new characterizations of 3-prime (3-semiprime) nearrings and hence for prime (semiprime) rings. These characterizations make 3-primeness looks like the forms of the other kinds of primeness. In fact, we show that a near-ring (a ring) is 3-prime (prime) if and only if $U V=\{0\}$ implies $U=\{0\}$ or $V=\{0\}$, where $U$ and $V$ are semigroup left ideals of $R$. Hence, a ring is prime if and only if it is without zero-semigroup right (left) ideal divisors. A similar result is made for 3-semiprime near-rings (semiprime rings) and we conclude that: for a near-ring $R$, if $r^{2} \neq 0$ for all $r \in R-\{0\}$, then $R$ is 3 -semiprime. We show that some kinds of near-rings are 3-prime if and only if they are 
2-prime. Also, we introduce a new kind of primeness in near-rings (the sixth one) called K-primeness and we show that it is totally different from the other kinds of primeness and it lies between 3-primeness and e-primeness. Depending on that, we give two chains of primeness in the class of zero-symmetric near-rings for comparison. In the last part of the paper, we study different kinds of prime ideals. We introduce a new kind of prime ideals called K-prime ideals and we show that they are different from the other kinds of prime ideals. they lie between 3-prime ideal and e-prime ideals. Also, we give a new characterization of 3-prime ideals and show that $P$ is a 3-prime ideal of $R$ if and only if $U V \subseteq P$ implies $U \subseteq P$ or $V \subseteq P$, where $U$ and $V$ are semigroup left ideals of $R$. We introduce new concepts called set-attractors, ideal-attractors, etc. which are generalizations of the new concepts above (set-divisors, etc.). We make a connection between these concepts and different kinds of prime ideals in near-rings to give a new characterization of these prime ideals. Finally, we use these concepts to show that: $P$ is a completely prime ideal of $R$ if and only if $R$ is without external $P$ set-attractors.

\section{On prime near-rings}

Let $R$ be a near-ring. It is clear that $R$ is without zero divisors if and only if $A B=\{0\}$ implies $A=\{0\}$ or $B=\{0\}$, where $A$ and $B$ are non-empty subsets of $R$. This observation gives us a hint of a new definition.

Definition 2.1. Let $R$ be a non-zero near-ring.

(1) Let $A$ be a non-empty subset of $R$. We say that $A$ is a left zero-set divisor (a right zeroset divisor) of $R$ if there exists a non-empty non-zero subset $B$ of $R$ such that $A B=\{0\}$ $(B A=\{0\})$. We say that $A$ is a zero-set divisor of $R$ if $A$ is a left or a right zero-set divisor of $R$.

(2) Let $A$ be an ideal of $R$. We say that $A$ is a left zero-ideal divisor (a right zero-ideal divisor) of $R$ if there exists a non-zero ideal $B$ of $R$ such that $A B=\{0\}(B A=\{0\})$. We say that $A$ is a zero-ideal divisor of $R$ if $A$ is a left or a right zero-ideal divisor of $R$.

We can do same definitions if $A$ is a left (right) ideal, a left (right) $R$-subgroup, a two-sided $R$-subgroup, a semigroup left (right) ideal or a semigroup ideal.

Definition 2.1 generalizes the concept of zero divisors in rings and near-rings. So, we have the following remark.

Remark 2.1. From Definition 2.1, we can rewrite the definitions of different kinds of the primeness as follows:

Let $R$ be a near-ring. Then

(1) $R$ is completely prime if and only if $R$ is without zero divisors if and only if $R$ is without zero-set divisors.

(2) $R$ is 0 -prime if and only if $R$ is without zero-ideal divisors.

(3) $R$ is 1-prime if and only if $R$ is without zero-right ideal divisors.

(4) $R$ is 2-prime if and only if $R$ is without zero-right $R$-subgroup divisors.

Remark 2.1 enhances a question: Can we get a definition of 3-primeness like that mentioned in Remark 2.1? The following result answers this question.

Theorem 2.1. Let $R$ be a near-ring. Then the following statements are equivalent:

(i) Ris 3-prime.

(ii) $a U=\{0\}$ implies $a=0$ or $U=\{0\}$, where $a \in R$ and $U$ is a semigroupleft ideal of $R$.
Primeness of near-rings 
AJMS

$26,1 / 2$

236

(iii) $A U=\{0\}$ implies $A=\{0\}$ or $U=\{0\}$, where $A$ is a non-empty subset of $R$ and $U$ is a semigroup left ideal of $R$. of $R$.

(iv) $U V=\{0\}$ implies $U=\{0\}$ or $V=\{0\}$, where $U$ and $V$ are semigroup left ideals

Proof. (i) implies (ii), (ii) implies (iii) and (iii) implies (iv) are clear.

To prove that (iv) implies (i), we will use the contradiction. For that purpose, suppose $R$ is not 3-prime. So there exist non-zero elements $x, y \in R$ such that $x R y=\{0\}$. Thus, $R x R y=\{0\}$. But $R x$ and $R y$ are semigroup left ideals of $R$, so $R x=\{0\}$ or $R y=\{0\}$ by (iv). Hence, $R\{0, x\}=\{0\}$ or $R\{0, y\}=\{0\}$ and either $\{0, x\}$ or $\{0, y\}$ is a semigroup left ideal of $R$. But $R$ is also a semigroup left ideal of $R$. Thus, $\{0, x\}=0,\{0, y\}=\{0\}$ or $R=\{0\}$ by (iv), a contradiction with that $x, y, R$ are all non-zero. So $R$ is 3-prime and (iv) implies (i).

For zero-symmetric near-rings, we have the following extra result.

Theorem 2.2. Let $R$ be a zero-symmetric near-ring. Then the following statements are equivalent:

(i) $R$ is 3-prime.

(ii) $U a=\{0\}$ implies $a=0$ or $U=\{0\}$, where $a \in R$ and $U$ is a semigroup right ideal of $R$.

(iii) $U A=\{0\}$ implies $U=\{0\}$ or, $A=\{0\}$ where $U$ is a semigroup right ideal of $R$ and $A$ is a non-empty subset of $R$. of $R$.

(iv) $U V=\{0\}$ implies $U=\{0\}$ or $V=\{0\}$, where $U$ and $V$ are semigroup right ideals

(v) $U V=\{0\}$ implies $U=\{0\}$ or $V=\{0\}$, where $U$ is a semigroup right ideal of $R$ and $V$ is a semigroup left ideal of $R$.

Now, we can add (5) to Remark 2.1:

(5) $R$ is 3-prime if and only if $U V=\{0\}$ implies $U=\{0\}$ or $V=\{0\}$, where $U$ and $V$ are semigroup left ideals of $R$ if and only if $R$ is without zero-semigroup left ideal divisors.

Since any ring is a zero-symmetric near-ring, we have the following result:

Corollary 2.3. A ring is prime if and only if it is without zero-semigroup right (left) ideal divisors.

Using the same idea, the following result gives us a result for 3-semiprime zero-symmetric near-rings.

Theorem 2.4. Let $R$ be a zero-symmetric near-ring. Then the following statements are equivalent:

(i) $R$ is 3-semiprime.

(ii) $a U=\{0\}$ implies $a=0$, where $a \in U$ and $U$ is a semigroup left ideal of $R$.

(iii) $U a=\{0\}$ implies $a=0$, where $a \in U$ and $U$ is a semigroup right ideal of $R$.

(iv) $U^{2}=\{0\}$ implies $U=\{0\}$, where $U$ is a semigroup left ideal of $R$.

(v) $U^{2}=\{0\}$ implies $U=\{0\}$, where $U$ is a semigroup right ideal of $R$.

Proof. (i) implies (ii). Suppose (i) holds. Let $U$ be a semigroup left ideal of $R$ such that $a U=\{0\}$, where $a \in U$. Then for all $v \in U$, we have $a R v=\{0\}$. Thus, $a R a=\{0\}$ and $a=0$ by (i). 
(i) implies (iii) can be proved by the same way.

(ii) implies (iv) and (iii) implies (v) are clear.

(iv) implies (v). Suppose that (iv) holds and $U^{2}=\{0\}$, where $U$ is a semigroup right ideal of $R$. So $u R u=\{0\}$ for all $u \in U$ and hence $R u R u=\{0\}$. But $R u$ is a semigroup left ideal of $R$. So $R u=\{0\}$ for all $u \in U$ by (iv). So $\{0, u\}$ is a semigroup left ideal of $R$ and $\{0, u\}\{0, u\}=\{0\}$ for all $u \in U$. So $u=0$ by (iv) and hence $U=\{0\}$.

(v) implies (i). Suppose that (v) holds and that $x R x=\{0\}$ for some $x \in R$. Thus, $x R x R=\{0\}$. But $x R$ is a semigroup right ideal of $R$, so $x R=\{0\}$ by (v). Hence, $\{0, x\}\{0, x\}=\{0\}$. But $\{0, x\}$ is a semigroup right ideal of $R$. Thus, $\{0, x\}=\{0\}$ by (v) and hence $x=0$. So $R$ is 3-semiprime and (v) implies (i).

Corollary 2.5. A ring $R$ is semiprime if and only if $U^{2}=\{0\}$ implies $U=\{0\}$, where $U$ is a semigroup right (left) ideal of $R$.

But in the general case of 3-semiprime near-rings, we have only the following result.

Theorem 2.6. Let $R$ be a near-ring. Then the following statements are equivalent:

(i) $R$ is 3-semiprime.

(ii) $a U=\{0\}$ implies $a=0$, where $a \in U$ and $U$ is a semigroup left ideal of $R$.

(iii) $U^{2}=\{0\}$ implies $U=\{0\}$, where $U$ is a semigroup left ideal of $R$.

Unfortunately, we cannot remove the word "zero-symmetric" in Theorems 2.2 and 2.4. The following example is the near-ring in [9, Appendix, E, 22] and it shows that the condition "zero-symmetric" in Theorems 2.2 and 2.4 is not redundant.

Example 1. Let $(R,+)$ be the Klein's four group $\{0, a, b, c\}$. Then it is an abelian group such that $x+x=0$ for all $x \in R$ and $x+y=z$ for all different non-zero elements $x, y, z \in R$. Define the multiplication on $R$ as follows:

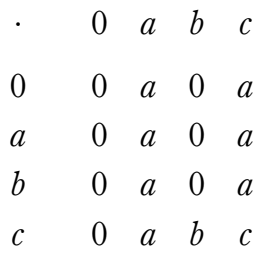

Clearly $R$ is an abelian non-zero-symmetric near-ring. The only semigroup right ideals of $R$ are $R,\{0, a\}$ and $\{0, a, b\}$. So $R$ satisfies the conditions " $U V=\{0\}$ implies $U=\{0\}$ or $V=\{0\}$, where $U$ and $V$ are semigroup right ideals of $R$ " and " $U^{2}=\{0\}$ implies $U=\{0\}$, where $U$ is a semigroup right ideal of $R^{\prime \prime}$. But $R$ is not 3-semiprime as $b R b=\{0\}$. From Theorem 2.6, we can deduce that there is a non-zero semigroup left ideal $V$ of $R$ such that $V^{2}=\{0\}$ and $v V=\{0\}$, where $v \in V-\{0\}$. It is easy to find out that $V=\{0, b\}$ and $v=b$.

From the above example, observe that

$$
\{0, a, b\} b=\{0, a, b\}\{b\}=\{0\} .
$$

So, we cannot use this example for (ii) or (iii) in Theorem 2.2 and for (iii) in Theorem 2.4. In fact, removing "zero-symmetric" from those parts is an open problem.

Corollary 2.7. Let $R$ be a near-ring. If $r^{2} \neq 0$ for all $r \in R-\{0\}$, then $R$ is 3-semiprime.

Proof. Suppose there exists a non-zero semigroup left ideal $U$ of $R$ such that $a U=\{0\}$, where $a \in U$. That means $a^{2}=0$. By hypothesis, $a=0$ and hence $R$ is 3 -semiprime.

Example 2. Let $R=\mathbb{Z}_{6}$. Then $R$ is semiprime since $r^{2} \neq 0$ for all $r \in R-\{0\}$.
Primeness of near-rings 
Example 3. Let $R=\{0,2,4,6,8,10,12\}$ the subring of $\mathbb{Z}_{14}$. Then $R$ is semiprime since $r^{2} \neq 0$ for all $r \in R-\{0\}$.

The converse of Corollary 2.7 is not true as the following example shows.

Example 4. Let $R=M_{2}\left(\mathbb{Z}_{2}\right)$. Then $R$ is a prime ring and hence semiprime, but

$$
\left[\begin{array}{ll}
0 & 1 \\
0 & 0
\end{array}\right]\left[\begin{array}{ll}
0 & 1 \\
0 & 0
\end{array}\right]=\left[\begin{array}{ll}
0 & 0 \\
0 & 0
\end{array}\right] .
$$

For commutative near-rings, we have the converse and we get the following result.

Corollary 2.8. Let $R$ be a commutative near-ring. Then $r^{2} \neq 0$ for all $r \in R-\{0\}$ if and only if $R$ is 3-semiprime.

We conclude this section by the following results about the relation between 2-primeness and 3-primeness. The fact that $R$ is 3 -prime implies $R$ is 2 -prime is well-known. The following results have the converse.

Theorem 2.9. Let $R$ be a zero-symmetric near-ring such that $2 R=\{0\}$. Then $R$ is 3-prime if and only if $R$ is 2-prime.

Proof. Suppose that $x R y=\{0\}$. Thus, $x R y R=\{0\}$. But $x R$ and $y R$ are right $R$-subgroups of $R$. So $x R=\{0\}$ or $y R=\{0\}$ as $R$ is 2-prime. Hence, $\{0, x\} R=\{0\}$ or $\{0, y\} R=\{0\}$ and then either $\{0, x\}$ or $\{0, y\}$ is a right $R$-subgroup of $R$. But $R$ is also a right $R$-subgroup of $R$. Thus, $\{0, x\}=0,\{0, y\}=\{0\}$ or $R=\{0\}$. Hence, $x=0$ or $y=0$ and $R$ is 3-prime.

Theorem 2.10 Any distributive near-ring $R$ is 3-prime if and only if it is 2-prime.

Proof. Suppose that $R$ is 2-prime and $x R y=\{0\}$ for some $x, y \in R$. So $x R y R=\{0\}$ and hence $x R=\{0\}$ or $y R=\{0\}$. So $A R=\{0\}$ or $B R=\{0\}$, where $A=\{n x \mid n \in \mathbb{Z}\}$ and $B=\{n y \mid n \in \mathbb{Z}\}$. So $A$ and $B$ are right $R$-subgroups of $R$ and hence $A=\{0\}$ or $B=\{0\}$. Therefore, $x=0$ or $y=0$ and $R$ is 3-prime.

\section{K-prime near-rings}

In this section, we will introduce a new kind of primeness of near-rings called K-primeness. Firstly, we will begin with the following result.

Theorem 3.1. Let $R$ be a ring. Then the following statements are equivalent:

(i) $R$ is prime.

(ii) for any $0 \neq a, x, y \in R$, xs $a=y$ ra for all $s, r \in R-\{0\}$ implies $x=y$.

Proof. A ring $R$ is prime if and only if it is equiprime, so we will use the definition of equiprimeness, i.e. for any $0 \neq a, x, y \in R, x c a=y c a$ for all $c \in R$ implies $x=y$.

(i) implies (ii) is clear.

(ii) implies (i). Suppose (ii) holds. If for all $c \in R$, $x c a=y c a$ for $0 \neq a, x, y \in R$, then $(x-y) c a=0=0 r a$ for all $c, r \in R$. So $x=y$ by (ii).

Part (ii) enhances the following definition for near-rings.

Definition 3.1. Let $R$ be a near-ring. We say that $R$ is K-prime if, for any $0 \neq a, x, y \in R$, $x s a=y r a$ for all $s, r \in R-\{0\}$ implies $x=y$.

As we mentioned before for rings, a ring is prime if and only if it is equiprime. So we have the following result.

Corollary 3.2. A ring $R$ is prime if and only if it is K-prime.

The following result shows that every K-prime near-ring is zero-symmetric 3-prime.

Theorem 3.3. Let $R$ be a K-prime near-ring. Then $R$ is zero-symmetric 3-prime. 
Proof. Firstly, we will show that $R$ is zero-symmetric. If $R$ is not zero-symmetric, then it has at least one non-zero constant element $c$ (see [8, Theorem 1.15). For different elements $x, y$ of $R$, we have that $x s c=y r c=c$ for all $s, r \in R-\{0\}$, a contradiction with the hypothesis. So $R$ is zero-symmetric. Now, suppose $x R y=\{0\}$ for some $x, y \in R$. So $x c y=0$ for all $c \in R$. If $y \neq 0$, then $x c y=0 r y$ for all $c, r \in R$. So $x=0$ from the hypothesis and hence $R$ is 3-prime.

In the case of near-rings, we have only that e-primeness implies K-primeness as shown in the proof of Theorem 3.1 (since an e-prime near-ring is zero-symmetric [10]). But the converse is not true as we will show in the next example. We will use the near-ring mentioned in [9, Appendix, F, 7] in the next example.

Example 5. Let $(R,+)$ be the cyclic group $\mathbb{Z}_{5}$ and define the multiplication on $R$ as follows:

\begin{tabular}{|c|c|c|c|}
\hline & 0 & 1 & \\
\hline & 0 & 0 & \\
\hline 1 & 0 & 1 & \\
\hline 2 & 0 & 2 & \\
\hline 3 & 0 & 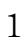 & \\
\hline & 0 & & \\
\hline
\end{tabular}

So $R$ is an abelian near-ring which is not a ring (as $(1+1) 2=3 \neq 4=2+2=(1) 2+(1) 2$ ). Clearly that $R$ is without zero divisors. Hence, $R$ is 3-prime. $R$ is not equiprime. Indeed, $1 c 1=3 c 1=c 1$ for all $c \in R$. But if $0 \neq a, x, y \in R$ such that $x s a=y r a$ for all $s, r \in R-\{0\}$, then $x=y$. Clearly that is true if $x$ or $y$ is equal to zero, since $R$ is without zero divisors. That is the only possible case. In fact, if $x s a=y r a$ for all $s, r \in R-\{0\}$ and $x, y, a$ are all non-zero, then from the table we can choose $s_{o}, r_{o} \in R-\{0\}$ to satisfy that $x s_{o}=1$ and $y r_{o}=2$. Hence, $a=2 a$ which implies that $a=0$ (from the table), a contradiction with $0 \neq a$. Therefore, K-primeness does not imply e-primeness.

Also, we can find zero-symmetric 3-prime near-rings which are not K-prime, as the following example shows.

Example 6. Let $R$ be a trivial zero-symmetric near-ring of order greater than 2. Clearly $R$ is 3prime. Taking two non-zero elements $x$ and $y$ such that $x \neq y$, we have $x s x=y r x=x$ for all $s, r \in R-\{0\}$. So $R$ is not K-prime.

Theorem 3.1, Theorem 3.3 and the examples after them show that K-primeness is a new kind of primeness.

Observe that K-primeness lies between 3-primeness and e-primeness (equiprimeness). So we have the following chain of primeness in the class of zero-symmetric near-rings:

The class of e-prime near-rings

$\subseteq$ The class of K-prime near-rings

$\subseteq$ The class of 3-prime near-rings

$\subseteq$ The class of 2-prime near-rings

$\subseteq$ The class of 1-prime near-rings

$\subseteq$ The class of 0 -prime near-rings

Remark 3.1. Observe that:

(i) It is well-known that $M_{o}(G)$ is e-prime (see [10]) and hence K-prime. Observe that it has zero divisors.

(ii) Since $M(G)$ is not zero-symmetric, so it is not K-prime (and hence not e-prime), but it has zero divisors.

Primeness of near-rings 
AJMS

$26,1 / 2$

(iii) Let $N$ be any near-field. Then $N$ is e-prime and hence K-prime. Indeed, for any $0 \neq a, x, y \in R$ such that $x c a=y c a$ for all $c \in R$, we have that $x=y$ by choosing $c=a^{-1}$. Observe that $N$ is without zero divisors.

(iv) Example 6 shows a 3-prime near-ring without zero divisors which is not K-prime (and hence not e-prime).

From the above parts in Remark 3.1, there is no relation between e-primeness (Kprimeness) and the existence of zero divisors in near-rings. So, we have another chain of the primeness in the class of zero-symmetric near-rings:

The class of completely prime near-rings

$\subseteq$ The class of 3-prime near-rings

$\subseteq$ The class of 2-prime near-rings

$\subseteq$ The class of 1-prime near-rings

$\subseteq$ The class of 0 -prime near-rings

\section{On prime ideals}

The next definition introduces K-prime ideals.

Definition 4.1. Let $R$ be a near-ring and $P$ an ideal of $R$. Then $P$ is a K-prime ideal of $R$ if for every $a \in R-P$ and $x, y \in R$, xra $-y s a \in P$ for all $r, s \in R-P$ implies $x-y \in P$.

Clearly $R$ is K-prime if and only if $\{0\}$ is a K-prime ideal of $R$.

The relationship between K-prime ideals and other kinds of prime ideals is stated in the following result.

Theorem 4.1. Let $R$ be a near-ring with an ideal $P$.

(i) If $P$ is a K-prime ideal of $R$, then $P$ is a 3-prime ideal of $R$.

(ii) If $P$ is an e-prime ideal of $R$, then $P$ is a K-prime ideal of $R$.

Proof. (i) Firstly, we will show that $P$ contains all the constant elements of $R$. Let $c$ be a constant element in $R$. If $c \in R-P$, then

$$
x r c-y s c=c-c=0 \in P
$$

for all $x, y \in R$ and $r, s \in R-P$. So $x-y \in P$ and hence $x-0=x \in P$ for all $x \in R$. Thus, $P=R$, a contradiction with $c \notin P$. So $c \in P$.

Now, suppose $a R b \subseteq P$ for some $a, b \in R$ and $b \notin P$. From above, any element $s \in R-P$ is a zero-symmetric element. So $0 s b=0 \in P$ for all $s \in R-P$. So $a r b-0 s b \in P$ for all $r, s \in R-P$. Thus, $a \in P$ by the hypothesis and $P$ is 3 -prime.

(ii) Firstly, observe that if $r \in P$ and $s \in R$ is a zero-symmetric element, then

$$
r s=(r+0) s-0 s \in P .
$$

Suppose $x r a-y s a \in P$ for all $r, s \in R-P$, where $a \in R-P$ and $x, y \in R$. So $x c a-y c a \in P$ for all $c \in R-P$. Now, suppose $c \in P$. As $a \notin P$, we have that $a$ is a zero-symmetric element (see [10]). So $c a \in P$ and hence $x c a-y c a \in P$. But $P$ is e-prime. So $x-y \in P$ and $P$ is a K-prime ideal of $R$.

The next result generalizes Theorem 2.1 for 3-prime ideals.

Theorem 4.2. Let $R$ be a near-ring and $P$ an ideal of $R$. Then the following statements are equivalent:

(i) $P$ is a 3-prime ideal of $R$.

(ii) $B U \subseteq P$ implies $B \subseteq P$ or $U \subseteq P$, where $B$ is a non-empty subset of $R$ and $U$ is a semigroup left ideal of $R$.

(iii) $U V \subseteq P$ implies $U \subseteq P$ or $V \subseteq P$, where $U$ and $V$ are semigroup left ideals of $R$. 
Proof. (i) implies (ii). Suppose (i) holds. Let $U$ be a semigroup left ideal of $R$ and $B$ be a nonempty subset of $R$ such that $B U \subseteq P$. If $B \nsubseteq P$, then there exists $b \in B-P$ such that $b R u \subseteq P$ for all $u \in U$. Thus, $U \subseteq P$ by (i).

(ii) implies (iii) is clear.

(iii) implies (i). To prove it, we will use the contradiction. Suppose that (iii) holds and $P$ is not a 3-prime ideal. So there exist $x, y R-P$ such that $x R y \subseteq P$. Thus, $R x R y \subseteq P$. So $R x \subseteq P$ or $R y \subseteq P$ by (iii). Hence, $R(P \cup\{x\}) \subseteq P$ or $R(P \cup\{y\}) \subseteq P$ and then $P \cup\{x\}$ or $P \cup\{y\}$ is a semigroup left ideal of $R$. But $R$ itself is also a semigroup left ideal of $R$. Thus, $P \cup\{x\} \subseteq P$, $P \cup\{y\} \subseteq P$ or $R \subseteq P$ by (iii), a contradiction with that $x, y \in R-P$. So $P$ is 3 -prime and (iii) implies (i).

Remark 4.1. From Theorem 4.2, a new characterization of 3-prime ideals can be written as follows:

(*) $P$ is a 3-prime ideal of $R$ if for every two semigroup left ideals $A$ and $B$ of $R, A B \subseteq P$ implies $A \subseteq P$ or $B \subseteq P$.

Using Theorem 4.2 and its proof, we can prove the following result which generalizes Theorem 2.2 for 3-prime ideals.

Theorem 4.3. Let $R$ be a zero-symmetric near-ring and $P$ an ideal of $R$. Then the following statements are equivalent:

(i) $P$ is a 3-prime ideal of $R$.

(ii) $U B \subseteq P$ implies $U \subseteq P$ or $B \subseteq P$, where $U$ is a semigroup right ideal of $R$ and $B$ is a non-empty subset of $R$.

(iii) $U V \subseteq P$ implies $U \subseteq P$ or $V \subseteq P$, where $U$ and $V$ are semigroup right ideals of $R$.

We cannot eliminate the condition "zero-symmetric" in Theorem 4.3 as the following example shows:

Example 7. Observe that $\{0\}$ is not a 3-prime ideal in Example 1 although it satisfies the condition "If $U V \subseteq\{0\}$, then $U \subseteq\{0\}$ or $V \subseteq\{0\}$, where $U$ and $V$ are semigroup right ideals of $R$ ". This shows that "zero-symmetric" in Theorem 4.3 is not redundant.

Now, we would like to generalize Definition 2.1.

Definition 4.2. Let $R$ be a near-ring with an ideal $I$.

(i) Let $A$ be a non-empty subset of $R$. We say that $A$ is a left $I$ set-attractor (a right $I$ set-attractor) of $R$ if there exists a non-empty subset $B$ of $R$ and $B \nsubseteq I$ such that $A B \subseteq I(B A \subseteq I)$. We say that $A$ is an $I$ set-attractor of $R$ if $A$ is a left or a right $I$ set-attractor of $R$.

(ii) Let $A$ be an ideal of $R$. We say that $A$ is a left $I$ ideal-attractor (a right $I$ ideal-attractor) of $R$ if there exists an ideal $B$ of $R$ and $B \nsubseteq I$ such that $A B \subseteq I(B A \subseteq I)$. We say that $A$ is an $I$ ideal-attractor of $R$ if $A$ is a left or a right $I$ ideal-attractor of $R$.

We can do the same definitions if $A$ is a left (right) ideal of $R$, a left (right, two-sided) $R$-subgroup of $R$, a semigroup ideal of $R$ or a semigroup left (right) ideal of $R$.

Example 8. Let $R$ be a near-ring with an ideal $I \neq R$. Any non-empty subset of $I$ is a right $I$ set-attractor of $R$ and hence an $I$ set-attractor of $R$. In particular, $I$ is an $I$ set-attractor of $R$. Also, if there exist an ideal (a left (right) ideal, a left $R$-subgroup, a semigroup left ideal) $B$ of $R$ such that $B \nsubseteq I$, then $I$ is an $I$ ideal-attractor ( $I$ left (right) ideal-attractor, $I$ left $R$-subgroupattractor, $I$ semigroup left ideal-attractor) of $R$.

Definition 4.3. Let $R$ be a near-ring with an ideal $P$. If $A$ is a $P$ set-attractor $(P$ idealattractor, etc.) of $R$, then we say that $A$ is an internal $P$ set-attractor ( $P$ ideal-attractor, etc.) of $R$

Primeness of near-rings 
AJMS

$26,1 / 2$

if $A \subseteq P$. If $A \nsubseteq P$, then we say that $A$ is an external $P$ set-attractor ( $P$ ideal-attractor, etc.) of $R$. If $R$ does not have any external $P$ set-attractors ( $P$ ideal-attractors, etc.), then we say that $R$ is without external $P$ set-attractors $(P$ ideal-attractors, etc.), i.e. for a $P$ set-attractor $(P$ idealattractor, etc.) $A$ of $R$, we have that $A \subseteq P$

Example 9. (i) Any near-ring $R$ is without external (or internal) $R$-set attractors.

(ii) Any near-ring without zero divisors is without external $\{0\}$-set attractors.

(iii) Let $R$ be the ring $\mathbb{Z}_{4}$. Take $P$ to be the ideal $\{0,2\}$. Then $R$ is without external $P$ set-attractors.

(iv) Let $R$ be the ring $\mathbb{Z}_{6}$. Take $P$ to be the ideal $\{0\}$. Then $\{2\},\{3\}$ and $\{4\}$ are external $P$ set-attractors and $\{0\}$ is an internal $P$ set-attractor.

Theorem 4.4. Let $R$ be a near-ring with an ideal $P$. Then the following statements are equivalent:

(i) $R$ is without external $P$ set-attractors.

(ii) $P$ is a completely prime ideal of $R$.

Proof. (i) implies (ii), Suppose (i) holds and $a b \in P$ for some $a, b \in R$. So $\{a\}\{b\} \subseteq P$. If $a \notin P$, then $b \in P$ by (i) and $P$ is completely prime.

(ii) implies (i). Suppose (ii) holds and $A$ is a $P$ set-attractor of $R$. So there exists a non-empty subset $B$ of $R$ and $B \nsubseteq P$ such that $A B \subseteq P$ or $B A \subseteq P$. Suppose the case is $A B \subseteq P$. Take $y \in B-P$. So $x y \in P$ for all $x \in A$ and then $A \subseteq P$ by (ii). By the same way we can do for the other case. So $R$ is without external $P$ set-attractors.

Remark 4.2. (i) If $I=\{0\}$ in Definition 4.2, then we have Definition 2.1.

(ii) From the above two definitions, Theorem 4.2 and 4.4, we can rewrite the statements of different kinds of prime ideals as follows:

Let $R$ be a near-ring with an ideal $P$. Then

(1) $P$ is completely prime if and only if $R$ is without external $P$ set-attractors if and only if for every two non-empty subsets $A$ and $B$ of $R, A B \subseteq P$ implies $A \subseteq P$ or $B \subseteq P$.

(2) $P$ is 0 -prime if and only if $R$ is without external $P$ ideal-attractors.

(3) $R$ is 1-prime if and only if $R$ is without external $P$ right ideal-attractors.

(4) $R$ is 2 -prime if and only if $R$ is without external $P$ right $R$-subgroup-attractors.

(5) $R$ is 3-prime if and only if $R$ is without external $P$ semigroup left ideal-attractors.

\section{References}

[1] G.L. Booth, N.J. Groenewald, Different Prime Ideals Innear-Rings. II. Rings and Radicals (Shijiazhuang, 1994), 131-140. in: Pitman Res. Notes Math. Ser., vol. 346, Longman, Harlow, 1996.

[2] G.L. Booth, N.J. Groenewald, S. Veldsman, A Kurosh-Amitsurprime radical for near-rings, Comm. Algebra 18 (9) (1990) 3111-3122.

[3] G. Ferrero, C. Cotti Ferrero, Nearrings, in: Some Developments linked to Semigroups and Groups, Kluwer Academic Publishers, Dordrecht, The Netherlands, 2002.

[4] N.J. Groenewald, Note on the completely prime radical innear-rings, in: Near-Rings and NearFields (Tübingen, 1985), 97-100, in: North-Holland Math. Stud., vol. 137, North-Holland, Amsterdam, 1987.

[5] N.J. Groenewald, Different prime ideals in near-rings, Comm. Algebra 19 (10) (1991) 2667-2675.

[6] W.L.M. Holcombe, Primitive Near-Rings (Doctoral dissertation), Uneversity of Leeds, 1970. 
[7] M. Holcombe, A hereditary radical for near-rings, Studia Sci. Math. Hungar. 17 (1-4) (1982) 453-456.

[8] J.D.P. Meldrum, Near-rings and their linkswith groups, in: Research Notes in Mathematics, vol. 134, Pitman (Advanced Publishing Program), Boston, MA, 1985.

[9] G. Pilz, Near-Rings. The Theory and its applications, second ed., in: North-Holland Mathematics Studies, vol. 23, North-Holland Publishing Co, Amsterdam, 1983.

[10] S. Veldsman, On equiprime near-rings, Comm. Algebra 20 (9) (1992) 2569-2587.

\section{Corresponding author}

Khalid H. Al-Shaalan can be contacted at: khshaalan@gmail.com

For instructions on how to order reprints of this article, please visit our website:

www.emeraldgrouppublishing.com/licensing/reprints.htm

Or contact us for further details: permissions@emeraldinsight.com 